\title{
BMJ Open Understanding income-related differences in distribution of child growth, behaviour and development using a cross-sectional sample of a clinical cohort study
}

\author{
Anne Fuller (1) , ${ }^{1,2,3}$ Arjumand Siddiqi, ${ }^{4,5}$ Faraz V Shahidi (D) ,4,6 \\ Laura N Anderson (1D ,2 Vincent Hildebrand, ${ }^{4,7}$ Charles D G Keown-Stoneman, ${ }^{4,8}$ \\ Jonathon L Maguire (D) ,,9 Catherine Birken (D) ,,3 On behalf of the TARGet Kids! \\ Collaborative
}

To cite: Fuller A, Siddiqi A, Shahidi FV, et al. Understanding income-related differences in distribution of child growth, behaviour and development using a crosssectional sample of a clinical cohort study. BMJ Open 2022;12:e056991. doi:10.1136/ bmjopen-2021-056991

- Prepublication history and additional supplemental material for this paper are available online. To view these files, please visit the journal online (http://dx.doi.org/10.1136/ bmjopen-2021-056991)

Received 31 August 2021 Accepted 19 January 2022

Check for updates

(C) Author(s) (or their employer(s)) 2022. Re-use permitted under CC BY-NC. No commercial re-use. See rights and permissions. Published by BMJ.

For numbered affiliations see end of article.

Correspondence to

Dr Anne Fuller;

anne.fuller@sickkids.ca

\section{ABSTRACT}

Objectives Children from low-income households are at an increased risk of social, behavioural and physical health problems. Prior studies have generally relied on dichotomous outcome measures. However, inequities may exist along the range of outcome distribution. Our objective was to examine differences in distribution of three child health outcomes by income categories (high vs low): body mass index (BMI), behaviour difficulties and development.

Design and setting This was a cross-sectional study using data from a primary care-based research network with sites in three Canadian cities, and 15 practices enrolling participants.

Participants, independent variable and outcomes The independent variable was annual household income, dichotomised at the median income for Toronto ( $<\$ C 80000$ or $\geq \$ C 80000$ ). Outcomes were: (1) growth (BMl z-score (zBMI) at 5 years, 1628 participants); (2) behaviour (Strengths and Difficulties Questionnaire (SDQ) at 3-5 years, 649 participants); (3) development (Infant Toddler Checklist (ITC) at 18 months, 1405 participants). We used distributional decomposition to compare distributions of these outcomes for each income group, and then to construct a counterfactual distribution that describes the hypothetical distribution of the low-income group with the predictor profile of the higher-income group.

Results We included data from 1628 (zBMI), 649 (SDQ) and 1405 (ITC) children. Children with lower family income had a higher risk distribution for all outcomes. For all outcomes, thecounterfactual distribution, which represented the distribution of children with lower-income who were assigned the predictor profile of the higherincome group, was more favourable than their observed distributions.

Conclusion Comparing the distributions of child health outcomes and understanding different risk profiles for children from higher-income and lower-income groups can offer a deeper understanding of inequities in child health outcomes. These methods may offer an approach that
Strengths and limitations of this study

- Large sample of young children in a major urban area in Canada.

- Use of distributional decomposition offers a novel alternative to simple regression for this population and these outcomes.

- All outcomes defined using objective measures or validated instruments relevant to clinical practice.

- Limits to generalisability related to lower proportion of children from lower income households and recruitment from primary care practices in an urban setting.

- Important predictors for each outcome may not have been included in this analysis.

can be implemented in larger datasets to inform future interventions.

\section{INTRODUCTION}

Income is an important determinant of child health, with children living in households from the lowest income quintile experiencing poorer health outcomes on multiple measures. ${ }^{1}$ Lower socioeconomic status, the broader construct that speaks to the material and social resources of families that are linked to income and education, has been associated with poorer child health outcomes across domains, ${ }^{2}$ including increased risk learning disability or serious behavioural difficulty, poorer educational outcomes ${ }^{3}$ and mental health challenges. ${ }^{4}$

There is a strong argument in favour of using continuous outcome measures in population health research. While population-level means or categorical definitions of outcomes may show improvement in important health 
outcomes over time, inequities may be overlooked by not examining the distributions of outcomes. ${ }^{5}$ Research findings based on categorised outcomes may be easier to use in clinical practice. However, studying continuous measures can reduce bias that may be introduced with assigning categories and may increase statistical power. ${ }^{6}$ Observing differences across the entire distribution may have important health implications but may not be captured in collapsed categories or using standard statistical tests due to smaller sample sizes at the tails of distributions or small but cumulatively important effect sizes. Understanding inequities in the full range of outcome distribution may also provide more nuanced findings to inform specific interventions. ${ }^{78}$

As research in the health sciences strives to generate evidence to support reducing inequities in child health, understanding inequities across the full range of outcome distribution may yield important knowledge that could inform specific targeted or population-level interventions, but may be overlooked using standard methods. However, research examining distributions in child health is extremely scarce. A scoping review exploring the literature assessing birth weight identified a conceptual rationale for studying inequities in distributions, but a gap in the use of distributions analytically in favour of categorical analyses such as quantile regression. ${ }^{9}$ Distributional decomposition is a method which has been used to explore inequities in distribution of outcomes in studies of health outcomes in adults, including body mass and blood pressure. ${ }^{710}$ This method offers an opportunity to observe differences between groups across the entire distribution of health outcomes, and then, by producing a counterfactual distribution of the outcomes by applying predictor profiles of one group to the other, to explore the ways in which possible predictors of the outcome may account for differences observed.

Obesity, mental illness and developmental delays are among the most significant chronic conditions faced by children and they share risk and protective factors, ${ }^{11} 12$ including poverty and childhood adversity. ${ }^{13}$ However, there is limited research examining income inequities in very young children, and data from populationbased clinical cohorts is scarce. Our first objective was to examine differences in the distribution of three child health outcomes in young children by income: body mass index (BMI), behaviour difficulties and development. Our second objective was to demonstrate a method called distribution decomposition which can be used to explore the extent to which differences between income groups across the outcome distribution can be accounted for by common predictors for each outcome.

\section{METHODS}

\section{Study design, setting and participants}

This was a cross-sectional study of children enrolled in the TARGet Kids! Research Network. TARGet Kids! is a primary care practice-based research network in the Greater Toronto Area and Kingston, Ontario, and Montreal, Quebec. Children less than 6 years old are recruited by trained research personnel embedded at primary care paediatric and family medicine practices. They are followed prospectively into adolescence. Participants complete standardised questionnaires and have anthropometrics measured at scheduled healthcare maintenance visits and are followed yearly. The sample used for this analysis includes outcomes collected from 2008 to 2019 . The study protocol and sample population have been described in detail. ${ }^{14}$

Exclusion criteria at enrolment are health conditions affecting growth, severe developmental delay, chronic health conditions (except asthma and high functioning autism), birth less than 32 weeks' gestation and families unable to complete questionnaires in English.

\section{Patient and public involvement}

The TARGet Kids! Research Network includes a Parent and Clinician Team which is actively involved in guiding the research directions and priorities of TARGet Kids!. ${ }^{5}$ Parents and patients were not actively involved in the design of this secondary analysis of existing TARGet kids! data. Results are disseminated to study participants through study communications and the TARGet Kids! website.

\section{Study assessments}

\section{Independent variable}

The independent variable was parent-reported annual household income. It is collected in the standardised TARGet Kids nutrition and health questionnaire with a single question, "what was your family income before taxes last year,' with 13 response categories, ranging from 'less than \$C10 000' to 'greater than \$C500000'. We created two categories, dichotomised at approximately the median household income in the Toronto Census Metropolitan Area based on the 2016 Canadian census $(<\$ C 80000$ or $\geq \$ C 80000)$. We dichotomiSed at the median income. ${ }^{16}$ We selected this cut point to represent a common measure of household income, and to ensure a robust sample size in both groups to permit the analysis.

\section{Dependent variables}

Dependent variables were: (1) growth (BMI z-score (zBMI) at 5 years); (2) child behaviour (total difficulties score on the Strengths and Difficulties Questionnaire (SDQ) at 3-5 years); (3) development (total score on the Infant Toddler Checklist (ITC) at 18 months).

To assess zBMI, height and weight were measured by trained research assistants according to standard protocols. ${ }^{17}$ BMI was calculated as weight in kilograms divided by squared heighted in metres and measured at 5 years old. Age and sex standardised zBMI was calculated using the recommended WHO growth standards. ${ }^{18}$

To assess child behaviour, we used the SDQ total difficulties score, measured between 3 and 5 years of age. The SDQ has been validated in children of all ages and across 
multiple countries and cultural groups. ${ }^{19}{ }^{20}$ The score is composed of 20 questions, and measures emotional problems, conduct problems, hyperactivity and peer problems. Higher score indicates greater difficulties.

To assess child development, we used the ITC (also known as the Communication and Symbolic Behaviour Scales: Developmental Profile), measured between 18 and 24 months. ${ }^{2122}$ This is a measure for clinical screening of social and communication developmental risk, validated for use between 6 and 24 months. Lower score indicates greater developmental risk.

\section{Covariates}

Child and maternal characteristics were used to produce predictor profiles. We selected these predictors to represent confounders commonly included in adjusted regression models and other analyses within the literature more broadly. For children, these were age (months), sex, birth weight (kilograms) and living arrangement (living with both parents or any other arrangement) for all models; gestational age (32-36 weeks, 37 weeks and greater) was included for ITC models only as an important predictor of development, ${ }^{23}$ and total months breastfed. For mothers, these were maternal age (years), education (high school or less, university or more), immigration status (born in Canada, born outside of Canada), ethnic ancestry (European/white, other) and BMI $\left(\mathrm{kg} / \mathrm{m}^{2}\right)$. Breastfeeding duration, and maternal BMI were included in the BMI models only as important predictors of child BMI. ${ }^{24}$

\section{Statistical analysis}

We used descriptive statistics to characterise the study population and describe the means and proportions of the outcomes of interest. We used Mann-Whitney U test and $\chi^{2}$ tests to compare predictors by income category. We used Kolmogorov-Smirnov tests to assess differences between distribution curves for each outcome. Using methods described by Siddiqi et $a l,{ }^{7}$ who adapted the DiNardo-Fortin-Lemieux decomposition, ${ }^{25}$ we then measured the distributional inequality. We first estimated the probability densities of each outcome for each income subgroup using an adaptative kernel estimator. We then calculated distributional inequality as the difference between the kernel density estimates of the two income subgroups. At any given point, it measures the difference between proportion of children in the lowerincome group and those in the higher income group. We depicted the kernel density distributions and the distributional inequality graphically.

We then proceeded with distributional decomposition separately for each outcome. Distributional decomposition offers a method to identify the proportion of inequality at each point in the outcome distribution that can be explained by a set of common predictors using a simple reweighting method originally developed by DiNardo $e t$ al. ${ }^{25}$ The syntax for this specific analysis using Stata was developed and refined by members of our team (V. Hildebrand). We estimated the counterfactual density function for each outcome of the lower-income group that would prevail were children in the lower-income group given the predictors of the higher income group. This involves reweighting the density function of the lowerincome group such that the reweighted sample of children in the lower-income group has the same predictors of the children in the higher income group. ${ }^{725}$ We then used the counterfactual weight to reweight the kernel density estimates to produce the counterfactual distribution. This counterfactual density distribution demonstrates how the observed distribution of the children in the lower-income group would change if they took on the predictor profile of children in the higher-income group. We plotted this reweighted counterfactual distribution to compare it visually to the original distributions for the higher-income and lower-income groups.

Because of smaller numbers of children at the high and low ends of the distributions of each variable for the lower-income group, we undertook a sensitivity analysis, reversing the reweighting by applying the predictor profile of the lower-income group to the higher-income group. This increases the likelihood of achieving 'common support', where all configurations of predictor profiles of the reweighted group are present in the reference group. We would expect the distribution to appear like the inverse of the first one.

As an additional analysis, to examine associations between income and each outcome, we also performed unadjusted and adjusted multinomial regression analyses. For zBMI, we used a four-category outcome based on clinical risk stratification and defined the variable as zBMI less than -2 , greater than or equal to -2 to 1 , greater than or equal to 1 to 2 , and greater than or equal to 2 . For zBMI, the reference group was set as the second category (normal weight status). For SDQ and ITC scores, we divided the total score into quartiles. For these outcomes, the reference group was set to the first quartile.

Statistical analyses were performed using Stata (V.14.2). ${ }^{26}$

\section{RESULTS}

For the BMI outcome 2123 children between 60 and 71 months had complete outcome and income reported, of whom 1628 ( $76 \%$ of total) had complete information for all variables and were included. For our SDQ cohort, 774 had complete outcome and income reported, 649 (84\% of total) of whom had complete information for each variable and were included. For our ITC cohort, 1698 had complete outcome and income reported, 1405 (81\% of total) of whom had complete information for each variable and were included (figure 1).

The predictor profiles of children from higher and lower-income households are shown in table 1. Children from lower-income households had a shorter duration of breastfeeding, had mothers who were younger; a lower proportion lived with both parents, had fewer mothers with a university education; a greater proportion had 
BMI Z-Score

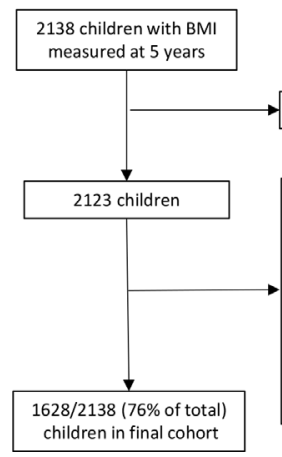

SDQ

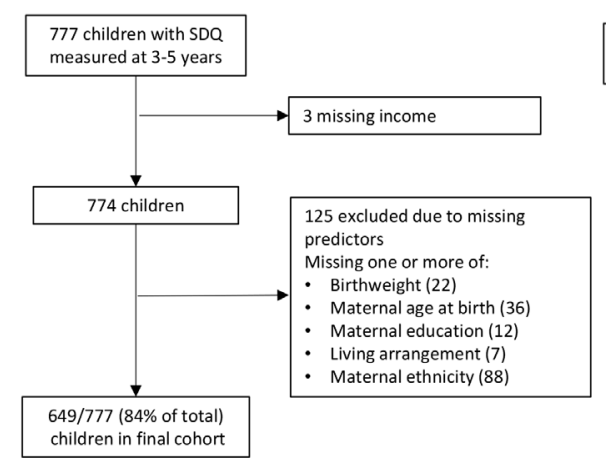

ITC

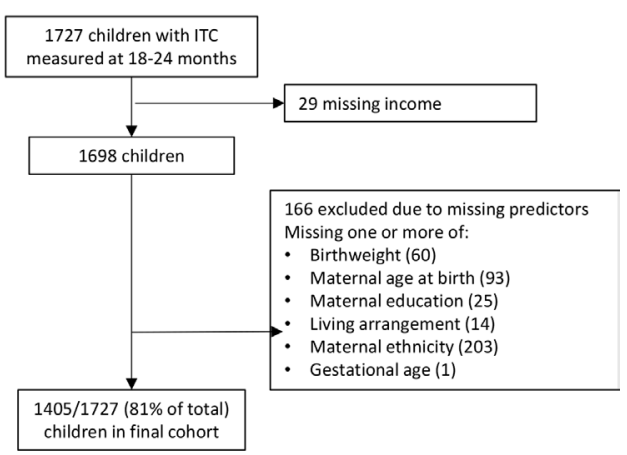

Figure 1 These flow diagrams show cohort definitions for each outcome and reasons for participant exclusion due to missing data. BMI, body mass index; ITC, Infant Toddler Checklist; SDQ, Strengths and Difficulties Questionnaire.

mothers who were immigrants to Canada or reported ethnic ancestry as other than European.

\section{Body mass index}

A greater proportion of children with higher income were in the normal weight category compared with children with lower-income $(84.9 \%$ vs $77.4 \%)$, while a greater proportion of children with low income were in the underweight, overweight, and obesity categories (table 1). Kolmogorov Smirnov Test (KST) test showed evidence of statistically significant difference between distributions income groups $(\mathrm{p}=0.004)$. Comparing the density distributions by income category, the distribution of children with high income was more concentrated around a zBMI of zero, while a higher proportion of children with low-income were at the tails of the distribution (figure 2A). Figure 2B shows the difference between the observed distributions.

When children from lower-income households were reweighted to have the predictor profiles of children from higher-income households, the distribution of zBMI within the normal range ( -1 to 1$)$ narrowed. This reweighted distribution is shown with the observed distributions in figure 2C. The residual, unexplained difference between the reweighted distribution and the higherincome distribution is shown in figure 2D. In this normal range, the difference between the re-weighted distribution for children from lower-income households and the distribution of children from higher-income households decreased substantially (figure 2D). However, at the tails of the distribution, the re-weighted distribution curve was largely unchanged from the observed distribution.

\section{Strengths and Difficulties Questionnaire}

Children from higher-income households had a lower mean SDQ score (7.2 vs 9.0) (table 1). KST test showed evidence of statistically significant difference between distributions income groups $(\mathrm{p}=0.002)$. Comparing the density distributions by income category, the differences in distribution were most notable in the lower and middle range of the score distribution, which had a lower proportion of children from lower-income households (figure 3A). There was a greater proportion of children from lower-income households in the high- risk range $(>17)$ as well. Figure 3B shows the difference between the observed distributions.

The reweighted distribution of SDQ total difficulties score for children from lower-income families in the lowrisk range shifted to the left, with a greater proportion having even lower scores than before. This reweighted distribution is shown with the observed distributions in figure 3C. The residual distribution had two peaks in the low-risk range, which were higher than the observed distribution for children from higher-income households, and a third peak in the high-risk range. The residual, unexplained difference between the re-weighted distribution and the high-income distribution is shown in figure 3D.

\section{Infant-toddler checklist}

Children from higher-income households had a higher mean ITC score indicating lower risk (46.6 vs 44.5) (table 1). KST test showed evidence of statistically significant difference between distributions income groups $(p<0.001)$. Comparing density distribution by income, the differences were notable across the distribution, with a greater proportion of children from lower-income households in the higher risk range (figure 4A). Figure 4B shows the difference between the observed distributions.

The reweighted distribution of ITC score for children from lower-income households shows that the distribution in the low-risk range (higher scores) is like the observed distribution from high-income households, indicating that common predictors explain much of the difference. This reweighted distribution is shown with the observed distributions in figure 4C. However, as total ITC score decreases into higher risk ranges, the reweighted distribution still shows a greater proportion of children from low-income households with lower scores. The residual, unexplained difference between the reweighted distribution and the high-income distribution is shown in figure $4 \mathrm{D}$.

\section{Sensitivity analyses}

Our sensitivity analysis, presented in online supplemental 1 , which reweighted the predictor profiles of children from higher-income households to have the predictor 


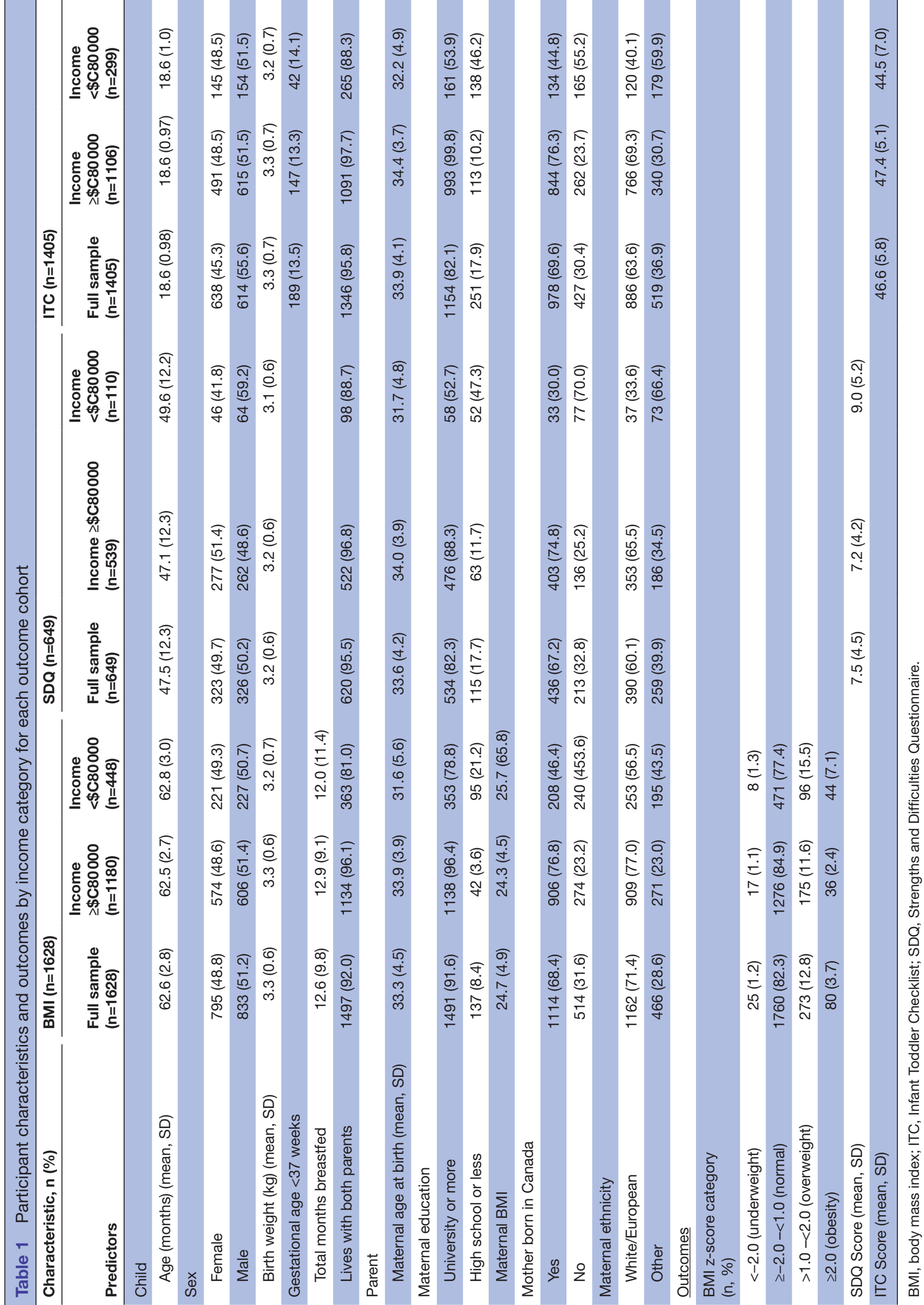


A

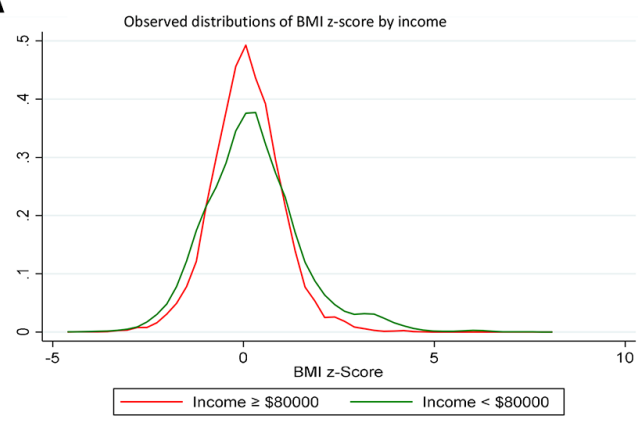

B

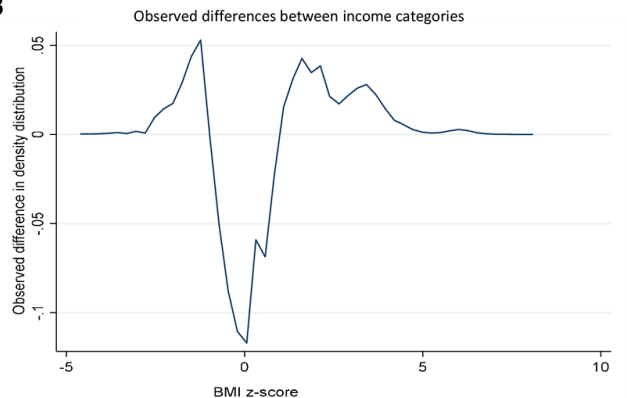

C

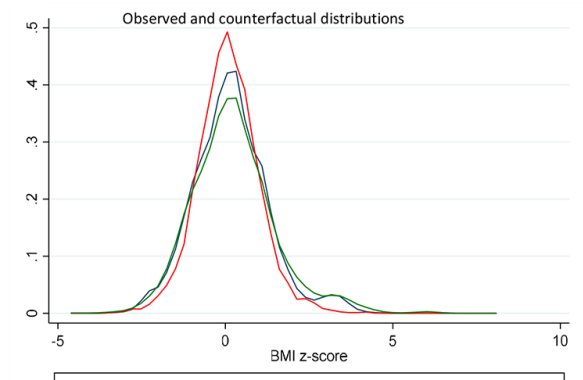

D
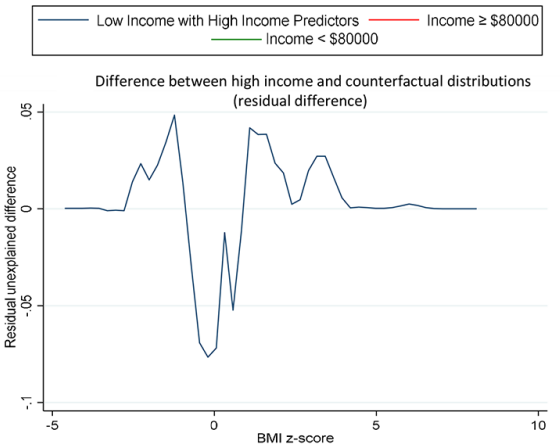

Figure 2 Distributions and distributional decomposition of BMI z-score, including observed distributions of BMI z-score by income (A); differences between observed distributions (B); observed distribution plus the counterfactual distribution of the low-income group with predictor profile of high-income group (C); and the residual difference between the high-income and counterfactual distributions (D). BMI, body mass index.

profile of children from lower-income households, showed a generally similar pattern in the low-risk range of the distribution for each outcome. Most notably, for SDQ, this analysis resolves the second peak of unexplained difference in the high-risk range, suggesting this may be due to low sample size in the lower-income group at the high end of the distribution.

Multinomial regression models for each outcome are found in online supplemental 2. The models generally demonstrate that lower income is associated with higher

C
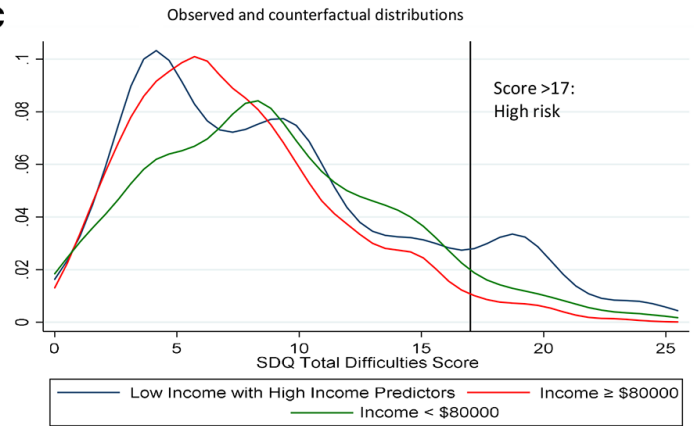

D Difference between high income and counterfactual distributions

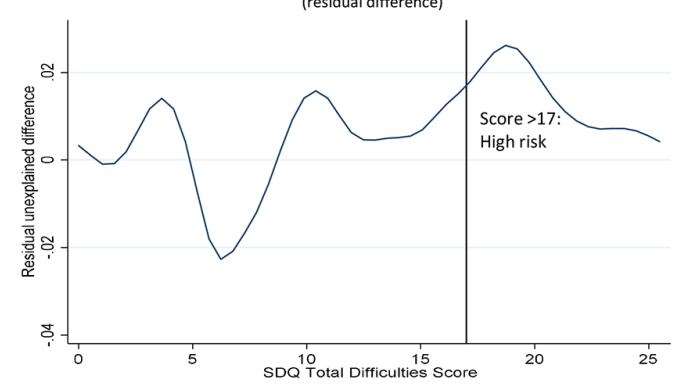

Figure 3 Distributions and distributional decomposition of SDQ Total Difficulties Score, including observed distributions of Total Difficulties Score by income (A); differences between observed distributions (B); observed distribution plus the counterfactual distribution of the low-income group with predictor profile of high-income group (C); and the residual difference between the high-income and counterfactual distributions (D). SDQ, Strengths and Difficulties Questionnaire. 
A

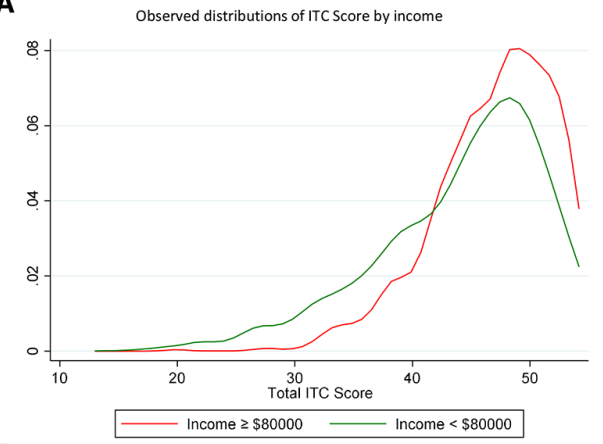

B

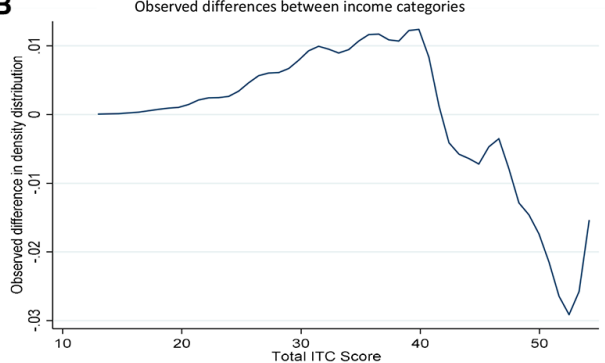

C

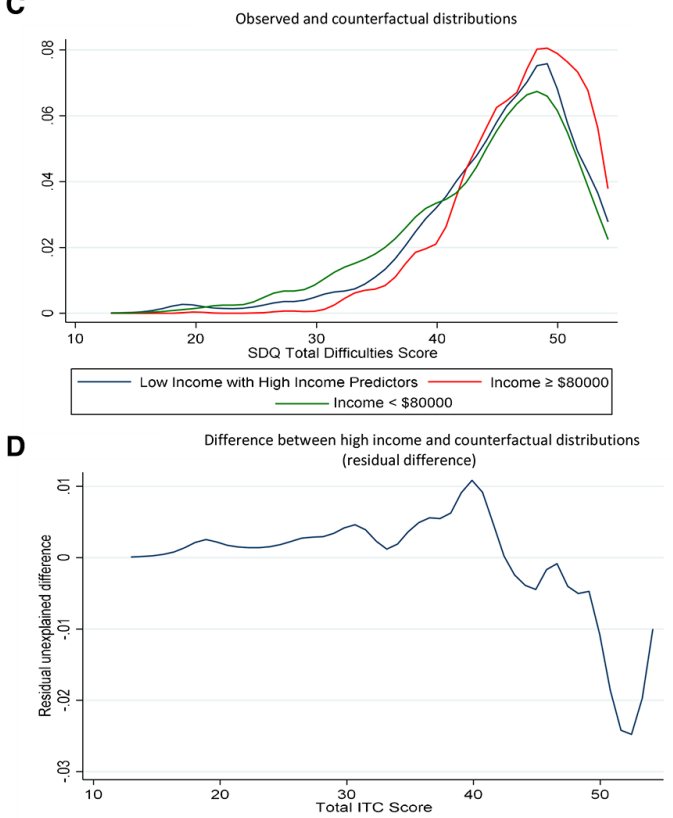

Figure 4 Distributions and distributional decomposition of total ITC Score, including observed distributions of ITC Score by income (A); differences between observed distributions (B); observed distribution plus the counterfactual distribution of the low-income group with predictor profile of high-income group (C); and the residual difference between the high-income and counterfactual distributions (D). ITC, Infant Toddler Checklist; SDQ, Strengths and Difficulties Questionnaire.

zBMI, higher SDQ Total Difficulties Score score, and lower ITC score. There was evidence of confounding by the covariates included.

\section{DISCUSSION}

In this study with a large cohort of young children, we found that there were notable differences in the distributions of children from higher-income and lower-income households for three important outcomes studied: zBMI, total behavioural difficulties and developmental risk, with a greater proportion of children with higher-income in the low-risk range of the distribution, and a greater proportion of those with lower-income in the higher risk range. When the distributions for children with lowerincome were reweighted to give them the predictor profiles of children with higher-income children, children with lower-income already in the low-risk range adopted a distribution that appeared to be even lower risk. After reweighting, children in the lower-income group with behavioural and developmental outcomes in the highrisk range adopted a distribution with a lower proportion of children at high risk. This was not the case for zBMI, where the reweighted distributions were like the observed distributions. Comparing observed distributions, the difference between income categories in the higher risk ranges (obesity, underweight) are smaller than the differences in the lower risk range (normal weight).

By comparing the observed distributions of continuous measures of child health by income, we can appreciate inequalities that may not be captured using categorical definitions that are used for clinical risk stratification.
Categorical measurement can collapse variation within each category, and this variation can yield important information. These inequalities may have clinical meaning; for example, small differences in SDQ score or in zBMI are related to differences in long-term behaviour and cardiometabolic outcomes, respectively. ${ }^{27}{ }^{28}$ Small differences in risk early in life may continue to grow through the life-course. For example, higher BMI in early life is associated with greater risk of obesity later. ${ }^{29}$ While the multinomial regression analyses generally support the differences observed in distributions, visualising the distributions offers a clearer picture of differences in the distribution, including transition points, for example, when distribution curves cross. Comparing distributions offers the opportunity to disaggregate differences that may not be appreciated with categorical outcome definitions.

The distributional decomposition analysis adds a further layer to our understanding of potential explanations for these inequities. For all outcomes, we found that the inequality between the observed distribution of children with higher-income and the counterfactual distribution was lower than the inequality between observed distributions of children within the 'low-risk' range of the distribution. However, in the higher-risk range, the counterfactual reduced the inequality to a variable degree depending on outcome. We suspect that the determinants of having clinically meaningful concerns about growth, behaviour or development are different than the determinants of where an individual falls in the lower risk range. For example, clinically significant behaviour difficulties on the SDQ may represent an underlying behaviour 
disorder such as attention-deficit disorder, while within the low-risk range, other factors such as parenting behaviours, which are more closely related to predictors in our predictor profiles, may be more influential.

For zBMI, the counterfactual distribution demonstrates that routine predictors of BMI explain some of the income-related inequality in the distribution within the normal range but does not explain the inequalities observed for children with obesity and underweight. It is possible that the determinants of obesity could be different than the determinants of underweight, ${ }^{30}$ or that low income is a primary driver of BMI. ${ }^{31} 32$

Compared with zBMI, routine predictors of child behaviour and mental health can explain more of the income-related inequality in the distribution of SDQ score, including at the higher range of the distribution. The highest risk range of the distribution may have represented children with significant morbidity, which likely has different predictors than a lower score. Our sensitivity analysis, which reweighted the children with highincome to have predictors of children with low-income, resolved this issue, suggesting sample size in the distribution of predictors for the lower-income group may be a contributor. The counterfactual distribution of the ITC was the closest to the observed distribution of children with higher-income of the three child health outcomes studied. It is possible that ITC had the strongest incomerelated predictors of the outcome included in the model, with parental education as a particularly important driver of parent-toddler communication, promoting language development. ${ }^{33}$

This study has several strengths. It includes a large sample of young children in a major urban area in Canada and employs a novel and revealing analysis. All outcomes were defined using objective measures (zBMI) orvalidated instruments (SDQ and ITC), which are relevant to clinical practice. This study also has certain limitations. Our sample had a lower proportion of children in the lower-income group, and particularly at the tail ends of distributions where there were fewer children overall, fewer children with each covariate pattern may have led to reduced robustness of the reweighted counterfactual. Future research could explore alterative categories of income. There was a smaller proportion of participants with certain characteristics which required categorisation of certain predictors and did not allow for stratification by potentially important predictors (eg, race/ethnicity). Children with missing data may come from households with low income or other stressors and are not represented. Furthermore, as our sample was drawn from a clinical setting, our recruitment and data collection process may have led to selection bias, with children from low-income families with poorer health over-represented compared with those with better health. This study is cross-sectional and causality cannot be inferred. Importantly, the relationship between income and health is likely bi-directional; while low-income may lead to poorer health outcomes, there is also evidence to suggest that chronic illness in childhood has adverse impacts on family income. ${ }^{34}$ One further consideration is the possibility that predictors of each outcome are also predictors of income (such as maternal education). In this case, some of the effects of income may actually be caused by these predictors. It is also likely that there are other meaningful predictors of each outcome that were not included in our predictor profile and may be important to the relationship between income and each outcome. For example, variables such as number of children in household, parenting styles and diet quality could be related to both income and outcome. Future research could explore a more detailed conceptual model of income-related predictors of each outcome to shed light on additional variables and incorporate longitudinal data to better understand causal relationships. Finally, the study takes place in primary care practices in a major urban area in Canada, participating families had higher income, were English-speaking, and may not be representative of children who lack access to primary care, live in rural areas, or who have other barriers to participation in a longitudinal study. Future research should seek out populations of children who are under-represented in these analyses.

\section{CONCLUSIONS}

This study examining income-related differences in child growth, behaviour and development found that there were differences in the distribution of each outcome between children from higher and lower-income families, with children from lower-income families showing a higher-risk profile. Common predictors of each outcome partially explained the inequality, most notably in the lowrisk range. These findings have important implications for health policies and interventions targeting income-based health inequities. Identifying that inequities likely have different predictors across the distribution suggests that future research should further explore predictor profiles that can explain income-related inequities in child health outcomes with a broader scope. It is possible that interventions to reduce inequities by addressing common predictors may improve outcomes in the low-risk range. However, targeted interventions addressing income specifically, as well as the circumstances experienced by families with low income, may be for those at high risk.

\section{Author affiliations}

${ }^{1}$ Pediatrics, The Hospital for Sick Children, University of Toronto, Toronto, Ontario, Canada

${ }^{2}$ Health Research Methods, Evidence and Impact, McMaster University, Hamilton, Ontario, Canada

${ }^{3}$ Child Health Evaluative Sciences, SickKids Research Institute, Toronto, Ontario, Canada

${ }^{4}$ Dalla Lana School of Public Health, University of Toronto, Toronto, Ontario, Canada ${ }^{5}$ Gillings School of Global Public Health, University of North Carolina, Chapel Hill, North Carolina, USA

${ }^{6}$ Institute for Work and Health, Toronto, Ontario, Canada

${ }^{7}$ Economics, York University - Glendon Campus, Toronto, Ontario, Canada

${ }^{8}$ Li Ka Shing Knowledge Institute, St Michael's Hospital, Toronto, Ontario, Canada

${ }^{9}$ Paediatrics, St Michael's Hospital, University of Toronto, Toronto, Ontario, Canada 
Acknowledgements We thank all participating children and families for their time and involvement in TARGet Kids! and are grateful to all practice site physicians, research staff, collaborating investigators, trainees, methodologists, biostatisticians, data management personnel, laboratory management personnel, and advisory committee members who are currently involved in the TARGet Kids! primary care practice-based research network.

Collaborators TARGet Kids! Research Network* TARGet Kids! Collaboration: Co-Leads: Catherine S. Birken, Jonathon L. Maguire; AdvisoryCommittee: Ronald Cohn, Eddy Lau, Andreas Laupacis, Patricia C. Parkin, Michael Salter, PeterSzatmari, Shannon Weir-Seeley; Science Review and Management Committees: Laura N. Anderson,Cornelia M. Borkhoff, Charles Keown-Stoneman, Christine Kowal, Dalah Mason; Site Investigators:Murtala Abdurrahman, Kelly Anderson, Gordon Arbess, Jillian Baker, Tony Barozzino, SylvieBergeron, Dimple Bhagat, Gary Bloch, Joey Bonifacio, Ashna Bowry, Caroline Calpin, DouglasCampbell, Sohail Cheema, Elaine Cheng, Brian Chisamore, Evelyn Constantin, Karoon Danayan, PaulDas, Mary Beth Derocher, Anh Do, Kathleen Doukas, Anne Egger, Allison Farber, Amy Freedman,Sloane Freeman, Sharon Gazeley, Charlie Guiang, Dan Ha, Curtis Handford, Laura Hanson, LeahHarrington, Sheila Jacobson, Lukasz Jagiello, Gwen Jansz, Paul Kadar, Florence Kim, Tara Kiran,Holly Knowles, Bruce Kwok, Sheila Lakhoo, Margarita Lam-Antoniades, Eddy Lau, Denis Leduc,Fok-Han Leung, Alan Li, Patricia Li, Jessica Malach, Roy Male, Vashti Mascoll, Aleks Meret, EliseMok, Rosemary Moodie, Maya Nader, Katherine Nash, Sharon Naymark, James Owen, Michael Peer,Kifi Pena, Marty Perlmutar, Navindra Persaud, Andrew Pinto, Michelle Porepa, Vikky Qi, NasreenRamji, Noor Ramji, Danyaal Raza, Alana Rosenthal, Katherine Rouleau, Caroline Ruderman, JanetSaunderson, Vanna Schiralli, Michael Sgro, Hafiz Shuja, Susan Shepherd, Barbara Smiltnieks, CinnthaSrikanthan, Carolyn Taylor, Stephen Treherne, Suzanne Turner, Fatima Uddin, Meta van den Heuvel,Joanne Vaughan, Thea Weisdorf, Sheila Wijayasinghe, Peter Wong, John Yaremko, Ethel Ying,Elizabeth Young, Michael Zajdman; Research Team: Farnaz Bazeghi, Vincent Bouchard, MarivicBustos, Charmaine Camacho, Dharma Dalwadi, Pamela Ruth Flores, Mateenah Jaleel, ChristineKoroshegyi, Tarandeep Malhi, Ataat Malick, Michelle Mitchell, Martin Ogwuru, Frank Ong, RejinaRajendran, Sharon Thadani, Julia Thompson, Laurie Thompson; Project Team: Mary Aglipay, ImaanBayoumi, Sarah Carsley, Katherine Cost, Karen Eny, Laura Kinlin, Jessica Omand, ShelleyVanderhout, Leigh Vanderloo; Applied Health Research Centre: Christopher Allen, Bryan Boodhoo,Olivia Chan, David W.H. Dai, Judith Hall, Peter Juni, Gurpreet Lakhanpal, Gerald Lebovic, KarenPope, Audra Stitt, Kevin Thorpe; Mount Sinai Services Laboratory: Rita Kandel, Michelle Rodrigues,Hilde Vandenberghe. Offord Centre for Child Studies Collaboration: Principal Investigator:Magdalena Janus; Co-investigator: Eric Duku; Research Team: Caroline Reid-Westoby, Patricia Raso,Amanda Offord.

Contributors AF conceptualised and designed the study, conducted the initial analyses and drafted the initial manuscript, and reviewed and revised the final manuscript. AS conceptualised and designed the study, reviewed the analyses and reviewed and revised the final manuscript. FVS and VH assisted with analysis, reviewed analyses, and reviewed and revised the final manuscript. LNA conceptualised and designed the study and reviewed and revised the final manuscript. CDGK-S managed study data, assisted with analysis, and reviewed and revised the final manuscript. JLM conceptualised and designed the study and reviewed and revised the final manuscript. CB conceptualised and designed the study, reviewed analyses, reviewed and revised initial manuscript drafts and the final manuscript and will act as guarantor for the manuscript. All authors approved the final manuscript as submitted and agreed to be accountable for all aspects of the work.

Funding Funding of the TARGet Kids! research network has been provided by the Canadian Institutes of Health Research (CIHR) Institute of Human Development, Child and Youth Health (PJT-168931), the SickKids Foundation and the St. Michael's Hospital Foundation. AF was supported by the Clinician-Scientist Training Programme through the SickKids Research Institute. AS is supported by the Canada Research Chair in Population Health Equity.

Disclaimer The funding agencies had no role in the design and conduct of the study, the collection, management, analysis and interpretation of the data, or the preparation, review and approval of the manuscript.

\section{Competing interests None declared.}

Patient consent for publication Not applicable.

Ethics approval This study was approved by the Research Ethics Board at the Hospital for Sick Children (REB \# 1000012436), Unity Health Toronto, and McGill University. Participants gave informed consent to participate in the study before taking part.
Provenance and peer review Not commissioned; externally peer reviewed.

Data availability statement Data may be obtained from a third party and are not publicly available. TARGet Kids! data are managed and analysed at the Applied Health Research Centre (AHRC) at the University of Toronto. Investigators whose proposed use of TARGet Kids! data has been approved by a research committee created for this purpose may access de-identified TARGet Kids! data.

Supplemental material This content has been supplied by the author(s). It has not been vetted by BMJ Publishing Group Limited (BMJ) and may not have been peer-reviewed. Any opinions or recommendations discussed are solely those of the author(s) and are not endorsed by BMJ. BMJ disclaims all liability and responsibility arising from any reliance placed on the content. Where the content includes any translated material, BMJ does not warrant the accuracy and reliability of the translations (including but not limited to local regulations, clinical guidelines, terminology, drug names and drug dosages), and is not responsible for any error and/or omissions arising from translation and adaptation or otherwise.

Open access This is an open access article distributed in accordance with the Creative Commons Attribution Non Commercial (CC BY-NC 4.0) license, which permits others to distribute, remix, adapt, build upon this work non-commercially, and license their derivative works on different terms, provided the original work is properly cited, appropriate credit is given, any changes made indicated, and the use is non-commercial. See: http://creativecommons.org/licenses/by-nc/4.0/.

\section{ORCID iDs}

Anne Fuller http://orcid.org/0000-0001-5930-5317

Faraz V Shahidi http://orcid.org/0000-0002-2789-1515

Laura N Anderson http://orcid.org/0000-0002-6106-5073

Jonathon L Maguire http://orcid.org/0000-0002-4083-8612

Catherine Birken http://orcid.org/0000-0003-0308-8645

\section{REFERENCES}

1 Neckerman KM, Garfinkel I, Teitler JO, et al. Beyond income poverty: measuring disadvantage in terms of material hardship and health. Acad Pediatr 2016;16:S52-9.

2 Oberg C, Colianni S, King-Schultz L. Child health disparities in the 21st century. Curr Probl Pediatr Adolesc Health Care 2016;46:291-312.

3 Chaudry A, Wimer C. Poverty is not just an indicator: the relationship between income, poverty, and child well-being. Acad Pediatr 2016;16:S23-9.

4 Fitzsimons E, Goodman A, Kelly E, et al. Poverty dynamics and parental mental health: determinants of childhood mental health in the UK. Soc Sci Med 2017;175:43-51.

5 Doyle YG, Furey A, Flowers J. Sick individuals and sick populations: 20 years later. J Epidemiol Community Health 2006;60:396-8.

6 Altman DG, Royston P. The cost of dichotomising continuous variables. BMJ 2006;332:1080.1.

7 Siddiqi A, Shahidi FV, Hildebrand V, et al. Illustrating a "consequential" shift in the study of health inequalities: a decomposition of racial differences in the distribution of body mass. Ann Epidemiol 2018;28:236-41.

8 Jones CP. Living beyond our "means": new methods for comparing distributions. Am J Epidemiol 1997;146:1056-66.

9 Ramraj C, Pulver A, O'Campo P, et al. A scoping review of socioeconomic inequalities in distributions of birth outcomes: through a conceptual and methodological lens. Matern Child Health $J$ 2020;24:144-52.

10 Basu S, Hong A, Siddiqi A. Using decomposition analysis to identify modifiable racial disparities in the distribution of blood pressure in the United States. Am J Epidemiol 2015;182:345-53.

11 Korczak DJ, Lipman E, Morrison K, et al. Are children and adolescents with psychiatric illness at risk for increased future body weight? A systematic review. Dev Med Child Neurol 2013;55:980-7.

12 Halfon N, Larson K, Slusser W. Associations between obesity and comorbid mental health, developmental, and physical health conditions in a nationally representative sample of US children aged 10 to 17. Acad Pediatr 2013;13:6-13.

13 Shonkoff JP, Garner AS, Committee on Psychosocial Aspects of Child and Family Health, et al. The lifelong effects of early childhood adversity and toxic stress. Pediatrics 2012;129:e232-46.

14 Carsley S, Borkhoff CM, Maguire JL, et al. Cohort profile: the applied Research Group for kids (target kids!). Int J Epidemiol 2015;44:776-88.

15 Lavigne M, Birken CS, Maguire JL, et al. Priority setting in paediatric preventive care research. Arch Dis Child 2017;102:748-53. 
16 Statistics Canada, 2016 Census of Population. Statistics Canada. 2017. Various geographies. census profile. 2016 census. statistics Canada Catalogue no. 98-316-X2016001. Ottawa. Released September 13, 2017.

17 de Onis M, Garza C, Onyango AW, et al. [WHO growth standards for infants and young children]. Arch Pediatr 2009;16:47-53.

18 WHO Multicentre Growth Reference Study Group. WHO child growth standards based on length/height, weight and age. Acta Paediatr Suppl 2006;450:76-85.

19 Stone LL, Otten R, Engels RCME, et al. Psychometric properties of the parent and teacher versions of the strengths and difficulties questionnaire for 4- to 12-year-olds: a review. Clin Child Fam Psychol Rev 2010;13:254-74.

20 Mieloo CL, Bevaart F, Donker MCH, et al. Validation of the SDQ in a multi-ethnic population of young children. Eur J Public Health 2014;24:26-32.

21 Wetherby AM, Brosnan-Maddox S, Peace V, et al. Validation of the Infant-Toddler checklist as a broadband screener for autism spectrum disorders from 9 to 24 months of age. Autism 2008;12:487-511.

22 Wetherby A, Prizant B. Communication and symbolic behavior scales developmental Profile- first Normed edition. Baltimore, MD: Paul H. Brookes, 2002

23 Woythaler M. Neurodevelopmental outcomes of the late preterm infant. Semin Fetal Neonatal Med 2019;24:54-9.

24 Ortega-García JA, Kloosterman N, Alvarez L, et al. Full breastfeeding and obesity in children: a prospective study from birth to 6 years. Child Obes 2018;14:327-37.
25 DiNardo J, Fortin NM, Lemieux T. Labor market institutions and the distribution of wages, 1973-1992: a semiparametric approach. Econometrica 1996;64:1001-44.

26 StataCorp. Stata statistical software: release 14. College Station, TX: StataCorp LP, 2015.

27 Goodman A, Goodman R. Strengths and difficulties questionnaire as a dimensional measure of child mental health. J Am Acad Child Adolesc Psychiatry 2009;48:400-3.

28 Kolsgaard MLP, Joner G, Brunborg C, et al. Reduction in BMI z-score and improvement in cardiometabolic risk factors in obese children and adolescents. The Oslo Adiposity Intervention Study a hospital/public health nurse combined treatment. BMC Pediatr 2011;11:47.

29 Nader PR, O'Brien M, Houts R, et al. Identifying risk for obesity in early childhood. Pediatrics 2006;118:e594-601.

30 Yanovski JA. Pediatric obesity. An introduction. Appetite 2015;93:3-12.

31 Gundersen C, Lohman BJ, Garasky S, et al. Food security, maternal stressors, and overweight among low-income US children: results from the National health and nutrition examination survey (19992002). Pediatrics 2008;122:e529-40.

32 Gundersen C, Mahatmya D, Garasky S, et al. Linking psychosocial stressors and childhood obesity. Obes Rev 2011;12:e54-63.

33 Hawa VV, Spanoudis G. Toddlers with delayed expressive language: an overview of the characteristics, risk factors and language outcomes. Res Dev Disabil 2014;35:400-7.

34 Kuhlthau K, Hill KS, Yucel R, et al. Financial burden for families of children with special health care needs. Matern Child Health $J$ 2005;9:207-18 